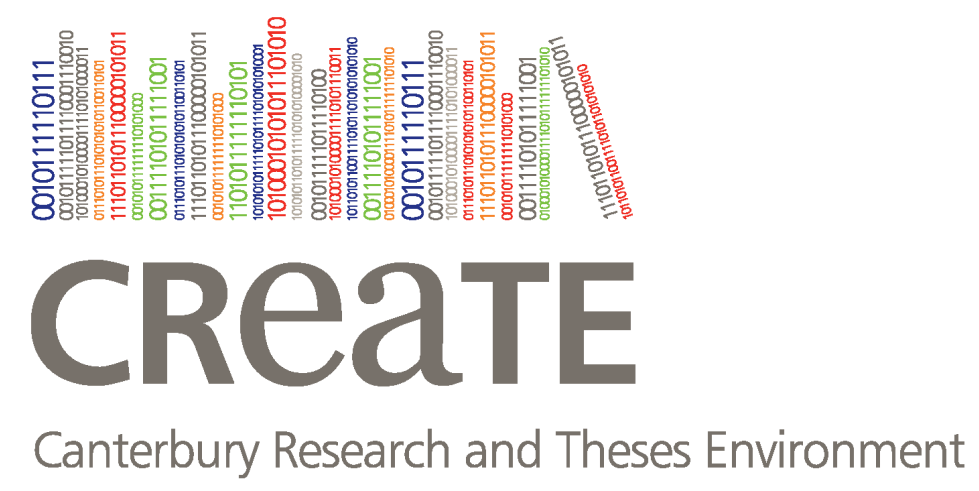

Canterbury Christ Church University's repository of research outputs http://create.canterbury.ac.uk

Please cite this publication as follows:

Beedie, C., Hurst, P., Coleman, D. A., Foad, A., Benedetti, F., Cohen, E., Davis, A., Elseworth-Edelsten, C., Flowers, E., Roelands, B., Hettinga, F., Raglin, J., Szabo, A., Camerone, E., Barbiani, D., Lane, A., Lindheimer, J., Schiphof-Godart, I. and Harvey, S.C. (2018) Consensus statement on placebo effects in sports and exercise: the need for conceptual clarity, methodological rigour, and the elucidation of neurobiological mechanisms. European Journal of Sport Science. ISSN 17461391.

Link to official URL (if available):

https://doi.org/10.1080/17461391.2018.1496144

This version is made available in accordance with publishers' policies. All material made available by CReaTE is protected by intellectual property law, including copyright law. Any use made of the contents should comply with the relevant law.

Contact: create.library@canterbury.ac.uk 


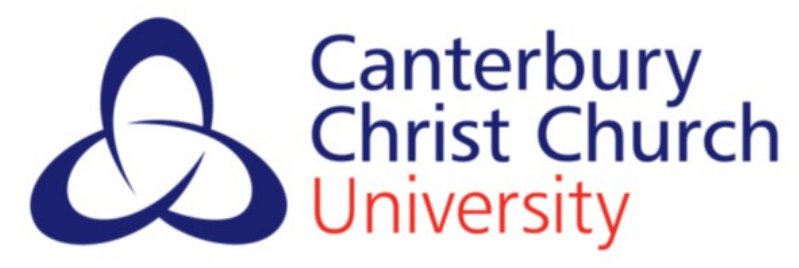




\title{
Consensus statement on placebo effects in sports and exercise: The need for conceptual clarity, methodological rigour, and the elucidation of neurobiological mechanisms.
}

\author{
Christopher Beedie ${ }^{1}$, Fabrizio Benedetti ${ }^{2}$, Diletta Barbiani ${ }^{2}$, Eleanora Camerone ${ }^{3}$, Emma \\ Cohen ${ }^{4}$, Damian Coleman ${ }^{1}$, Arran Davis ${ }^{4}$, Charlotte Edelsten ${ }^{1}$, Elliott Flowers ${ }^{5}$, Abby Foad ${ }^{1}$, \\ Simon Harvey ${ }^{1}$, Florentina Hettinga ${ }^{5}$, Philip Hurst ${ }^{6}$, Andrew Lane ${ }^{7}$, Jacob Lindheimer ${ }^{8}$, John \\ Raglin ${ }^{9}$, Bart Roelands ${ }^{10}$, Lieke Schiphof-Godart ${ }^{11}$, Attila Szabo ${ }^{12}$

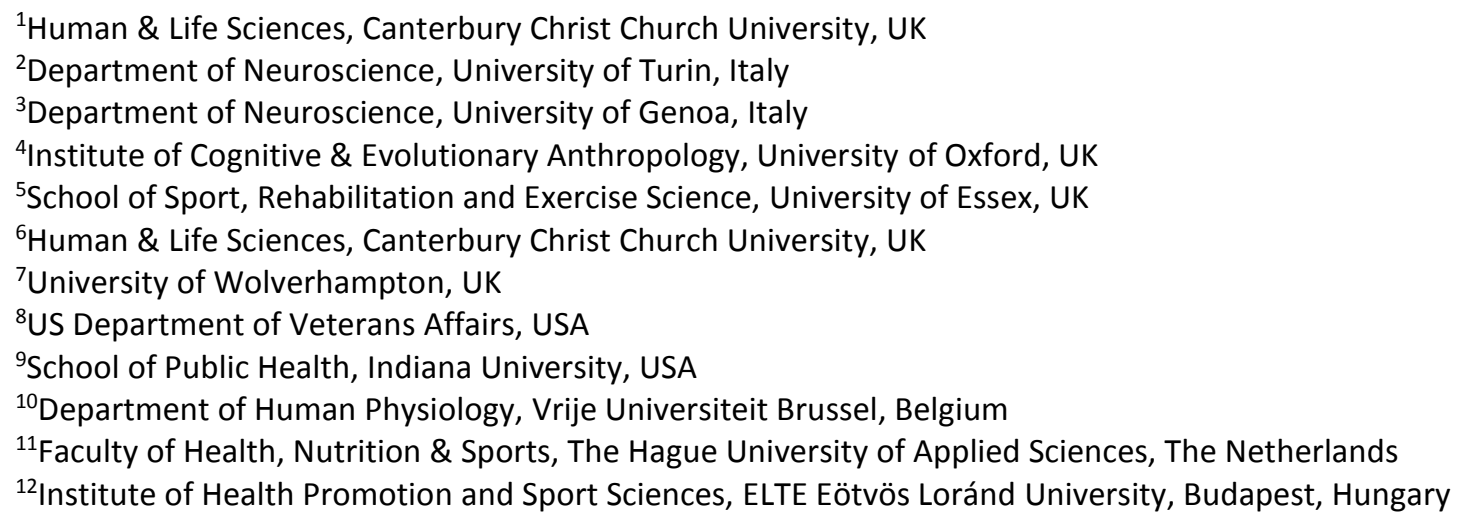

\section{Abstract}

In June 2017 a group of experts in anthropology, biology, kinesiology, neuroscience, physiology, and psychology convened in Canterbury, UK, to address questions relating to the placebo effect in sport and exercise. The event was supported exclusively by Quality Related (QR) funding from the Higher Education Funding Council for England (HEFCE). The funder did not influence the content or conclusions of the group. No competing interests were declared by any delegate.

During the meeting and in follow-up correspondence, all delegates agreed the need to communicate the outcomes of the meeting via a brief consensus statement. The two specific aims of this statement are to encourage researchers in sport and exercise science to:

1. Where possible, adopt research methods that more effectively elucidate the role of the brain in mediating the effects of treatments and interventions.

2. Where possible, adopt methods that factor for and/or quantify placebo effects that could explain a percentage of inter-individual variability in response to treatments and intervention

\section{Keywords}

Neuroscience, nutrition, performance, psychology. 


\section{Background}

In June 2017 a group of experts in anthropology, biology, kinesiology, neuroscience, physiology, and psychology convened in Canterbury, UK, to address questions relating to the placebo effect in sport and exercise. The event was supported exclusively by Quality Related (QR) funding from the Higher Education Funding Council for England (HEFCE). The funder did not influence the content or conclusions of the group. No competing interests were declared by any delegate. Jacob Lindheimer is with the US Department of Veterans Affairs. The contents of this paper do not represent the views of the Department of Veterans Affairs or of the United States Government.

\section{List of attendees}

Diletta Barbiani (Neuroscience, University of Turin, Italy); Christopher Beedie (Sport Psychology, Canterbury Christ Church University, UK. Organiser); Fabrizio Benedetti (Neuroscience, University of Turin, Italy); Eleanora Camerone (Neuroscience, Kings College, London); Emma Cohen (Anthropology, University of Oxford, UK); Damian Coleman (Physiology, Canterbury Christ Church University, UK); Arran Davis (Anthropology, University of Oxford, UK); Charlotte Edelsten (Physiology, Canterbury Christ Church University, UK); Elliott Flowers (Psychology, University of Essex, UK); Abby Foad (Psychology, Canterbury Christ Church University, UK); Simon Harvey (Evolutionary Biology, Canterbury Christ Church University, UK); Florentina Hettinga (Physiology, University of Essex, UK); Philip Hurst (Psychology, University of Birmingham, UK); Andrew Lane (Psychology, University of Wolverhampton, UK); Jacob Lindheimer (Kinesiology, US Department of Veterans Affairs, USA); John Raglin (Kinesiology, Indiana University, USA); Bart Roelands (Neuroscience, Free University of Brussels, Belgium); Lieke Schiphof-Godart (The Hague University of Applied Sciences, The Netherlands); Attila Szabo (Psychology, ELTE Eötvös Loránd University, Budapest, Hungary).

During the meeting and in follow-up correspondence, all delegates agreed the need to communicate the outcomes of the meeting via a brief consensus statement. The two specific aims of this statement are to encourage researchers in sport and exercise science to:

1. Where possible, adopt research methods that more effectively elucidate the role of the brain in mediating the effects of treatments and interventions.

2. Where possible, adopt methods that factor for and/or quantify placebo effects that could explain a percentage of inter-individual variability in response to treatments and interventions.

The contents of this statement were derived through two days of round-table discussion and by correspondence over the subsequent months. Although this document is not a comprehensive review of the literatures in question, we contend that the consensus statement is of value to scientists and practitioners given the broad expertise of the membership of the group. We are cognizant that given the state of the literature related to placebo effects in the sport and exercise literature, some may regard this statement to be 
premature. However, we believe that findings elsewhere render the piece both timely and important. Specifically, the absence of clear trends or theoretical frameworks in placebo effect research in sport and exercise has led to variations in definitions and concepts, which have constrained advances in knowledge. Our goal is to present recommendations built on what is known about placebo effects in a broad range of scientific disciplines, including anthropology, biology, medicine, neuroscience and psychology. By doing so we feel this will provide both a broader consensus of basic concepts and increase awareness of methodological challenges to facilitate advances in knowledge. This awareness and the resulting recommendations will have impacts on future sport and exercise studies, as they inform rigorous study protocols, and the interpretation of findings.

\section{Context}

The placebo effect. The placebo effect is a desirable outcome resulting from a person's expected and/or learned response to a treatment or situation. A nocebo effect is an undesirable outcome resulting from similarly learned or expected cues. Given that placebo effects are common in response to drugs, researchers in a broad range of disciplines, most notably medicine, have attempted to control for them for almost 80 years, with the first placebo controlled clinical trial reported in 1944 (Chalmers \& Clarke, 2004). However, whilst the placebo effect has been controlled for almost ubiquitously in medicine since that study, research into the placebo effect itself was slow to develop.

In a seminal review (Beecher, 1955), the significant influence of the placebo effect in a range of medical conditions was reported. Since then, and specifically since the mid-1990s, there has been mounting evidence for the clinically meaningful contribution of placebo effects (Kirsch \& Sapirstein, 1998), and for unambiguous and consistent neurophysiological pathways underlying these effects (Wager \& Atlas, 2015). This evidence has led many researchers to position the placebo condition in research as an active treatment, as opposed to either a passive control or a collective term for a range of non-specific effects such as regression to the mean (Finniss, Kaptchuk, Miller, \& Benedetti, 2010).

Placebo effects in sport and exercise. Reviews of the sport and exercise literature have reported beneficial effects associated with the administration of placebo treatments (Beedie \& Foad, 2009; Bérdi, Köteles, Szabó, \& Bárdos, 2011). Most published studies in sport focused on the effects of placebo nutritional supplements, including caffeine, creatinemonohydrate, carbohydrate and even anabolic steroids. Whilst some studies reported the effects of the placebo against no treatment (Ariel \& Saville, 1972; Beedie, Coleman, \& Foad, 2007), others directly compared the effects of the placebo with the substance which the placebo was described to be (Clark, Hopkins, Hawley, \& Burke, 2000; McClung \& Collins, 2007). The majority of these studies reported positive effects of placebos on objective measures of performance outcome and subjective markers of physiological stress such as rating of perceived exertion (Duncan, 2010). Fewer studies have included objective markers of physiological stress such as blood lactate, oxygen uptake and heart rate (Foad, Beedie, \& Coleman, 2008). Reviews of this literature (Beedie \& Foad, 2009; Bérdi et al., 2011) indicate the findings of sport placebo research are generally consistent with those reported in other 
scientific disciplines such as medicine, psychiatry and neuroscience (for review see Pollo, Carlino, \& Benedetti, 2011). These can be summarized as follows:

1. Placebo effects in relation to baseline/no-treatment conditions are frequently observed in studies in which the placebo condition is treated as an experimental manipulation (Beedie \& Foad, 2009; Bérdi et al., 2011). In short, when researchers and practitioners treat the placebo as a manipulation, they realise that standard control conditions are often not really controls.

2. Effects are often of a magnitude that is meaningful to the athlete. That is, they are associated with a likely beneficial effect on performance or performance-related variables (Batterham \& Hopkins, 2006; Beedie, Stuart, Coleman, \& Foad, 2006).

3. In many cases placebo effects constitute a significant percentage of the overall effect of the treatment the placebo purports to be, for example caffeine (Foad et al., 2008).

4. Placebo effects constitute approximately $50 \%$ of the effects of exercise on both subjective (i.e., anxiety, depression, energy, fatigue) and/or objective (i.e., cognitive tasks) psychological outcomes (Lindheimer, O’Connor, \& Dishman, 2015).

Causes of placebo effects. Researchers in neuroscience, medicine and psychology have examined causes of placebo effects, and much of this work has focused on environmental cues of conditioning and/or expectation. Conditioning can be either conscious or unconscious learning resulting from the repeated pairing of a stimulus treatment and a response. Research on human performance reported in the neuroscience literature demonstrates effects of conditioned placebo responses of morphine (Benedetti, Pollo, \& Colloca, 2007) and caffeine (Pollo, Carlino, \& Benedetti, 2008) on muscle performance. Expectation is conceived as a conscious cognitive process often resulting from verbal instruction. Work in sport and exercise science to date has generally adopted experimental designs based on expectation. Saunders et al. (2017) reported that the performance of subjects who, when asked post-test, identified correctly that they had ingested caffeine, improved to a greater degree than did that of subjects who had ingested caffeine but did not believe this to be the case. Hence, knowingly ingesting caffeine increases performance more than ingesting caffeine unknowingly. Furthermore, the same authors reported that the performance of subjects who believed they had ingested caffeine but who had actually ingested placebo improved compared to those who correctly identified that they had ingested placebo, the latter associated with performance decrements in some subjects.

Neurobiological mechanisms of placebo effects. In arguably a landmark study (Levine, Gordon, \& Fields, 1978) it was demonstrated that the placebo effect on pain could be reversed by administration of the opiate antagonist naloxone. This finding suggested not only that the placebo effect in this context had a clear neurobiological mechanism, but that the mechanism of placebo analgesia was what would be expected with a real analgesic treatment. Since this publication, an extensive body of research has shown not only that expectation and conditioning independently cause placebo effects, but that each operate via discrete neurophysiological pathways. In fact, this research has shown that there is not just one mechanism of the placebo effect, but several, each operating across different 
neurobiological pathways. For example, in a study of pain (Amanzio \& Benedetti, 1999), the placebo effects were either experimentally conditioned, or resulted from experimentally induced expectations (conditioning was achieved by means of either the opioid agonist morphine hydrochloride, or the nonopioid ketorolac tromethamine). Expectation resulted in placebo responses that were completely blocked by the opioid antagonist naloxone, suggesting that the placebo effect in question was a function of opioid pathways. Expectation cues together with morphine conditioning also produced placebo responses that were completely blocked by naloxone, as did morphine conditioning alone. By contrast, ketorolac conditioning together with expectation cues elicited a placebo effect that was only partially blocked by naloxone, and ketorolac conditioning alone produced placebo responses that were naloxone-insensitive. Therefore, Amanzio and Benedetti elicited different types of placebo responses using different methods of induction, conditioning and expectation. These data, and findings of many other studies (for review see Benedetti \& Dogue, 2015) indicate that placebo effects can be intact neurophysiological events, the mechanisms of which vary in relation to contextual cues, ultimately affecting activity in endogenous selfregulatory systems. These are proposals that could, and perhaps should, be tested in sport and exercise, especially given that many of the variables examined in placebo effect research in neuroscience and elsewhere, such as pain, fatigue, and oxygen availability, have implications for both.

The nocebo effect. Evidence suggests that the nocebo effect, an undesirable effect resulting from anticipated or conditioned negative outcomes, is also underpinned by neurobiological pathways (Tracey, 2010). For example, negative verbal suggestions induce anticipatory anxiety, which is associated with the activation of cholecystokinin (CCK) which in turn, facilitates pain transmission. Analogous to work in endogenous opioids and naloxone cited above, CCK-antagonists have been found to block this anxiety-induced hyperalgesia (Benedetti, Lanotte, Lopiano, \& Colloca, 2007). The implications of these effects can be significant and long-lasting, with some studies reporting that just one experience of a nocebo effect can influence the efficacy of future treatments (Colloca \& Miller, 2011). In the context of sport and exercise science, only a handful of studies have investigated the nocebo effect (Beedie et al., 2007; Bottoms, Buscombe, \& Nicholettos, 2014; Hurst, Foad, \& Beedie, 2017). While ethical constraints may limit how much we can learn about this phenomenon, the evidence from neuroscience suggests it can significantly impair sport performance and negatively impact exercise outcomes. It is pertinent that research in sport has shown performance outcomes to fall below baseline in conditions where participants believed that the treatment had been withheld, for example when participants are informed or suspect they have been assigned to the control condition (Beedie et al., 2006; Foad et al., 2008). (We recognise that such a scenario may not represent an "active" nocebo. In other words, being denied treatment may worsen performance by a different mechanism than a nocebo effect, where the participant believes the substance to be harmful.)

Context and placebo responses. Placebo and nocebo effects encompass a wide range of phenomena that are not restricted to a direct response to the administration of a placebo/nocebo treatment. The results obtained in both the placebo and treatment arms of a drug trial comprise all the influences associated with administration of that drug. These 
can include but are not limited to: expectation, previous experiences, the interaction between participant and researcher, trust, empathy and the ritual surrounding the administration. In the clinical or research setting, ambiguous language such as "this stuff might work" is likely far less effective than definitive language such as "6mg per kg body weight of caffeine has been found to improve 40-km time trial performance by between 2 and $3 \%$ among most competitive cyclists studied". Furthermore, such instructions delivered by a senior professor might be more effective than when delivered by a first-year graduate student. A wealth of data in social psychology describes contextual factors that impact on treatment outcomes, some of which is reported in sport and exercise literature (Beedie, Foad, \& Hurst, 2015; Trojian \& Beedie, 2008). Collectively these and the neurophysiological mechanisms described above and elsewhere have been described as 'the new physiology of the doctor patient relationship' (Benedetti, 2013).

\section{Statement of problem}

The placebo effect is likely a factor in all sport and exercise research studies involving human participants, even in those with a placebo control condition. However, as we have indicated above, placebo and nocebo effects can be complex. For example, all other things being equal, in a research study or in the field, the performance of athletes or exercisers with positive expectations of a treatment would theoretically improve to a greater degree than the performance of those with either no expectation or negative expectation. Likewise, the performance of athletes who have habitually used a treatment - and who therefore might exhibit conditioned responses to that treatment - should in theory be enhanced to a greater degree than the performances of athletes not conditioned to that particular treatment (which of course may contest ideas related to reduced effectiveness resulting from habituation, Bell \& McLellan, 2002; Goldstein et al., 2010). Given what is known about placebo effects, it is not unreasonable to suggest that the placebo responsiveness of any one person might be a factor in their responsiveness to a wide range of biologically active treatments. In this context, recent research indicated that athletes who used or intended to use sport supplements were more placebo responsive than those that do not (Hurst et al., 2017). However, in most experimental designs currently used, researchers would be unaware of these effects.

\section{Recommendations}

At our meeting we agreed that future sport and exercise research could be enhanced by considering the possibility that placebo and nocebo effects might explain a significant percentage of outcome variance. Further, that research would benefit by incorporating variables that facilitate the assessment of the placebo contribution to overall treatment effects. We arrived at consensus on the following recommendations, all of which we believe are critical in enhancing knowledge in relation to the interactions between brain and body in sport and exercise. In proposing these recommendations, we are not proposing they are only relevant to increasing understanding of placebo and nocebo effects, in many respects each represents good practice in a wide range of research contexts beyond placebo research. 
1. Use of no-treatment groups alongside placebo and treatment groups. Such designs recognise the research cited above that position the placebo condition as an active treatment as opposed to a passive control. Elucidating such effects would not only improve the validity and reliability of research (under-performance among controls has the potential to falsely inflate the apparent effect size, whilst large placebo effects compared to baseline might raise questions regarding the biological effectiveness of the treatment), but also help us understand the effects of expectation and/or conditioning on treatment outcomes that might enhance practice.

2. Assessment of conditioning. Researchers should use standardised measures of participants' experience relating to the treatment under examination. This could help researchers identify the degree to which learned/acquired responses have influenced outcomes. This is especially important in efficacy trials when previous experiences of a treatment may lead to biased samples and incorrect conclusions.

3. Assessment of expectation. Researchers should examine subjects' perceptions of the treatment, for example their belief in the effect of the treatment and the degree to which they are certain to have benefited from it (and therefore did not ingest a placebo). A measure such as the Stanford Expectation of Treatments Scale (Younger, Gandhi, Hubbard, \& Mackey, 2012) could be adapted to this context. Measures of expectation could assist in distinguishing placebo responders from placebo nonresponders and add to the explained variance in findings.

4. Examination of mechanisms. Researchers should conduct more mechanistic research to clarify direct and placebo treatment effects. For example, the ergogenic effects of caffeine on performance have been replicated using placebo caffeine, but questions remain as to whether there are mechanisms common to both placebo and real caffeine. Ingested caffeine can exert neurophysiological effects (Kaasinen, Aalto, Någren, \& Rinne, 2004) as can caffeine that is tasted but not ingested (De Pauw et al., 2015). Caffeine can also elicit metabolic effects (Hodgson, Randell, \& Jeukendrup, 2013). It is possible that the effects of ingested caffeine on performance might be identical to those of ingested placebo caffeine, or different, for example metabolic in the case of real caffeine and neurobiological in the case of placebo caffeine (De Pauw et al., 2015; Kaasinen et al., 2004).

5. Examination of patterns in variability. Researchers should investigate large standard deviations in response to treatments, specifically the possibility that these could be hiding relatively homogeneous sub-groups of placebo responders and placebo nonresponders. The proportion of people responding to a placebo varies from study to study. While there may not be a single setting in which every individual would respond to a placebo, findings from conditioning-based placebo research suggest that the capacity to respond to a placebo is perhaps more than a binary and stable personality trait.

6. Consideration and measurement of contextual factors. As mentioned above, researchers should recognise that placebo and nocebo effects encompass a wide range of effects that are not just direct effects from the administration of a placebo. All such factors need to be considered in study design and data analysis. Certainly, 
such factors should be standardised where possible; for example, a treatment administered by one experimenter might differ systematically in effectiveness from the same treatment administered by another. In this context, fine details of participant contact, communication and treatment should be reported in studies.

7. Consideration and measurement of potentially overlapping causes. Researchers should consider the likely overlap of effects in sport and exercise. For example, the apparent enhanced performance resulting from false positive feedback in a pacing study might theoretically be explained by motivational effects that would be evident in increased effort and increased physiological stress, or by placebo effects driven by neurobiological reward mechanisms that might not be observed in increased physiological stress. Likewise, administration of a placebo might enhance confidence that could itself result in a more effective pacing strategy and improved performance. Researchers should not only keep an open mind as to potentially overlapping mechanisms, but should seek wherever possible to measure those that might be implicated. Certainly, it should not be assumed that because the intervention was aimed at enhancing motivation or confidence, observed effects on performance are de facto the result of motivation or confidence only.

\section{References}

Amanzio, M., \& Benedetti, F. (1999). Neuropharmacological dissection of placebo analgesia: Expectation-activated opioid systems versus conditioning-activated specific subsystems. The Journal of Neuroscience, 19(1), 484-494.

Ariel, G., \& Saville, W. (1972). Anabolic steroids. The physiological effects of placebos. Medicine and Science in Sports, 4(2), 124-126.

Batterham, A. M., \& Hopkins, W. G. (2006). Making meaningful inferences about magnitudes. International Journal of Sports Physiology and Performance, 1(1), 50-57. doi:10.1123/ijspp.1.1.50

Beecher, H. K. (1955). The powerful placebo. Journal of the American Medical Association, 159(17), 1602-1606.

Beedie, C., Foad, A., \& Hurst, P. (2015). Capitalizing on the placebo component of treatments. Current Sports Medicine Reports, 14(4), 284-287. doi:10.1249/JSR.0000000000000172

Beedie, C. J., Coleman, D. A., \& Foad, A. J. (2007). Positive and negative placebo effects resulting from the deceptive administration of an ergogenic aid. International Journal of Sport Nutrition and Exercise Metabolism, 17(3), 259-269.

Beedie, C. J., \& Foad, A. J. (2009). The placebo effect in sports performance: A brief review. Sports Medicine, 39(4), 313-329. doi:10.2165/00007256-200939040-00004

Beedie, C. J., Stuart, E. M., Coleman, D. A., \& Foad, A. J. (2006). Placebo effects of caffeine on cycling performance. Medicine and Science in Sports and Exercise, 38(12), 2159-2164. doi:10.1249/01.mss.0000233805.56315.a9

Bell, D. G., \& McLellan, T. M. (2002). Exercise endurance 1, 3, and $6 \mathrm{~h}$ after caffeine ingestion in caffeine users and nonusers. Journal of Applied Physiology, 93(4), 1227-1234.

Benedetti, F. (2013). Placebo and the new physiology of the doctor-patient relationship. Physiological Reviews, 93(3), 1207-1246. doi:10.1152/physrev.00043.2012 
Benedetti, F., \& Dogue, S. (2015). Different placebos, different mechanisms, different outcomes: Lessons for clinical trials. PLoS One, 10(11), e0140967. doi:10.1371/journal.pone.0140967

Benedetti, F., Lanotte, M., Lopiano, L., \& Colloca, L. (2007). When words are painful: Unraveling the mechanisms of the nocebo effect. Neuroscience, 147(2), 260-271. doi:10.1016/j.neuroscience.2007.02.020

Benedetti, F., Pollo, A., \& Colloca, L. (2007). Opioid-mediated placebo responses boost pain endurance and physical performance: Is it doping in sport competitions? The Journal of Neuroscience, 27, 11934-11939. doi:10.1523/JNEUROSCI.3330-07.2007

Bérdi, N., Köteles, F., Szabó, A., \& Bárdos, G. (2011). Placebo effects in sport and exercise. A meta-analysis. European Journal of Mental Health, 6, 196-212. doi:10 5708/EJMH 6201125.

Bottoms, L., Buscombe, R., \& Nicholettos, A. (2014). The placebo and nocebo effects on peak minute power during incremental arm crank ergometry. European Journal of Sport Science, 14(4), 362-367. doi:10.1080/17461391.2013.822564

Chalmers, I., \& Clarke, M. (2004). Commentary: The 1944 patulin trial: The first properly controlled multicentre trial conducted under the aegis of the British Medical Research Council. International Journal of Epidemiology, 33(2), 253-260. doi:10.1093/ije/dyh162

Clark, V., Hopkins, W., Hawley, J., \& Burke, L. (2000). Placebo effect of carbohydrate feedings during a 40-km cycling time trial. Medicine and Science in Sports and Exercise, 32(9), 1642-1647.

Colloca, L., \& Miller, F. G. (2011). The nocebo effect and its relevance for clinical practice. Psychosomatic Medicine, 73(7), 598-603. doi:10.1097/PSY.0b013e3182294a50

De Pauw, K., Roelands, B., Knaepen, K., Polfliet, M., Stiens, J., \& Meeusen, R. (2015). Effects of caffeine and maltodextrin mouth rinsing on P300, brain imaging, and cognitive performance. Journal of Applied Physiology, 118(6), 776-782. doi:10.1152/japplphysiol.01050.2014

Duncan, M. (2010). Placebo effects of caffeine on anaerobic performance in moderately trained adults. Serbian Journal of Sports Sciences, 4(3), 99-106.

Finniss, D. G., Kaptchuk, T. J., Miller, F., \& Benedetti, F. (2010). Placebo effects: Biological, clinical and ethical advances. Lancet, 375(9715), 686-695. doi:10.1016/S0140-6736(09)61706-2

Foad, A. J., Beedie, C. J., \& Coleman, D. A. (2008). Pharmacological and psychological effects of caffeine ingestion in 40-km cycling performance. Medicine and Science in Sports and Exercise, 40(1), 158-165. doi:10.1249/mss.0b013e3181593e02

Goldstein, E. R., Ziegenfuss, T., Kalman, D., Kreider, R., Campbell, B., Wilborn, C., . . Antonio, J. (2010). International society of sports nutrition position stand: Caffeine and performance. Journal of the International Society of Sports Nutrition, 7(1), 5. doi:10.1186/1550-2783-7-5

Hodgson, A. B., Randell, R. K., \& Jeukendrup, A. E. (2013). The metabolic and performance effects of caffeine compared to coffee during endurance exercise. PLOS ONE, 8(4), e59561. doi:10.1371/journal.pone.0059561 
Hurst, P., Foad, A. J., \& Beedie, C. (2017). Athletes intending to use sports supplements are more likely to respond to a placebo. Medicine and Science in Sports and Exercise, 49(9), 1877-1883. doi:10.1249/MSS.0000000000001297

Kaasinen, V., Aalto, S., Någren, K., \& Rinne, J. O. (2004). Expectation of caffeine induces dopaminergic responses in humans. European Journal of Neuroscience, 19(8), 23522356. doi:10.1111/j.1460-9568.2004.03310.x

Kirsch, I., \& Sapirstein, G. (1998). Listening to Prozac but hearing placebo: A meta-analysis of antidepressant medication. Prevention \& Treatment, 1(2). doi:10.1037/1522-3736.1.1.12a

Levine, J., Gordon, N., \& Fields, H. (1978). The mechanism of placebo analgesia. The Lancet, 312(8091), 654-657. doi:10.1016/S0140-6736(78)92762-9

Lindheimer, J. B., O'Connor, P. J., \& Dishman, R. K. (2015). Quantifying the placebo effect in psychological outcomes of exercise training: A meta-analysis of randomized trials. Sports Medicine, 45(5), 693-711. doi:10.1007/s40279-015-0303-1

McClung, M., \& Collins, D. (2007). "Because I know it will!": placebo effects of an ergogenic aid on athletic performance. Journal of Sport and Exercise Psychology, 29(3), 382394. doi:10.1123/jsep.29.3.382

Pollo, A., Carlino, E., \& Benedetti, F. (2008). The top-down influence of ergogenic placebos on muscle work and fatigue. European Journal of Neuroscience, 28(2), 379-388. doi:10.1111/j.1460-9568.2008.06344.x

Pollo, A., Carlino, E., \& Benedetti, F. (2011). Placebo mechanisms across different conditions: from the clinical setting to physical performance. Philosophical Transactions of the Royal Society B: Biological Sciences, 366(1572), 1790-1798. doi:10.1098/rstb.2010.0381

Saunders, B., de Oliveira, L. F., da Silva, R. P., de Salles Painelli, V., Goncalves, L. S., Yamaguchi, G., ... Gualano, B. (In press). Placebo in sports nutrition: A proof-ofprinciple study involving caffeine supplementation. Scandinavian Journal of Medicine and Science in Sports. doi:10.1111/sms.12793

Tracey, I. (2010). Getting the pain you expect: Mechanisms of placebo, nocebo and reappraisal effects in humans. Nature Medicine, 16(11), 1277-1283. doi:10.1038/nm.2229

Trojian, T. H., \& Beedie, C. J. (2008). Placebo effect and athletes. Current Sports Medicine Reports, 7(4), 214-217. doi:10.1249/JSR.0b013e31817ed050

Wager, T. D., \& Atlas, L. Y. (2015). The neuroscience of placebo effects: Connecting context, learning and health. Nature Reviews Neuroscience, 16, 403-418. doi:10.1038/nrn3976

Younger, J., Gandhi, V., Hubbard, E., \& Mackey, S. (2012). Development of the Stanford Expectations of Treatment Scale (SETS): A tool for measuring patient outcome expectancy in clinical trials. Clinical Trials, 9(6), 767-776. doi:10.1177/1740774512465064 